\title{
Integral Inequalities of Hermite-Hadamard Type for $r$-Convex Functions
}

\author{
Lingxiong Han, Guofeng Liu \\ College of Mathematics, Inner Mongolia University for Nationalities, Tongliao, China \\ Email: hlx2980@163.com
}

Received September 17, 2012; revised October 17, 2012; accepted October 25, 2012

\begin{abstract}
The main aim of this present note is to establish three new Hermite-Hadamard type integral inequalities for $r$-convex functions. The three new Hermite-Hadamard type integral inequalities for $r$-convex functions improve the result of original one by Hölder's integral inequality, Stolarsky mean and convexity of function.
\end{abstract}

Keywords: Hermite-Hadamard Integral Inequality; $r$-Convex Function; Logarithmic Mean; Stolarsky Mean

\section{Introduction}

The inequalities

$$
f\left(\frac{a+b}{2}\right) \leq \frac{1}{b-a} \int_{a}^{b} f(t) \mathrm{d} t \leq \frac{f(a)+f(b)}{2},
$$

which discovered by $\mathrm{C}$. Hermite and Hadamard for all convex functions $f:[a, b] \rightarrow(-\infty,+\infty)$ are known in the literature as Hermite-Hadamard inequalities.

We note that Hermite-Hadamard inequalities may be regarded as a refinement of the concept of convexity and they follows easily from Jenson's inequality. HermiteHadamard inequalities for convex functions has received renewed attention in recent years and a remarkable variety of refinements and generalizations have been found in [1-6].

Let $f^{p}(x), g^{q}(x)$ be integrable functions on

$$
[a, b], p, q>0, \frac{1}{p}+\frac{1}{q}=1,
$$

then the well known Hölder's integral inequality is given as

$$
\begin{aligned}
& \int_{a}^{b} f(x) g(x) \mathrm{d} x \\
& \leq\left(\int_{a}^{b} f^{p}(x) \mathrm{d} x\right)^{1 / p} \cdot\left(\int_{a}^{b} g^{q}(x) \mathrm{d} x\right)^{1 / q} .
\end{aligned}
$$

The following definition is well known in the literature.

Definition 1.1. Suppose

$$
f: I \subseteq(-\infty, \infty) \rightarrow(-\infty, \infty)
$$

If following inequality holds

$$
f(t x+(1-t) y) \leq t f(x)+(1-t) f(y)
$$

for any $x, y \in I$ and $t \in[0,1]$, then we say $f$ is convex function on $I$.

In [1], C. E. M. Pearce, J. Pecaric and V. Simic introduced the definition of $r$-convex function and studied the inequalities of Hermite-Hadamard type for $r$-convex functions.

Definition 1.2. ([1]) A function

$$
f:[a, b] \subseteq[0, \infty) \rightarrow(0, \infty)
$$

is said to be $r$-convex function on $[a, b]$, if

$$
\begin{aligned}
& f(t x+(1-t) y) \\
& \leq\left\{\begin{array}{cc}
{\left[t f^{r}(x)+(1-t) f^{r}(y)\right]^{1 / r}} & \text {, if } r \neq 0, \\
f^{t}(x) f^{1-t}(y), & \text { if } r=0 .
\end{array}\right.
\end{aligned}
$$

holds for any $x, y \in[a, b]$ and $t \in[0,1]$.

We have that 0 -convex functions are simply log-convex functions and 1-convex functions are ordinary convex functions.

The integral power mean $M_{p}$ (see [2]) of a positive function $f$ on $[a, b]$ is a functional given by

$$
M_{p}(f)=\left\{\begin{array}{l}
\left(\frac{1}{b-a} \int_{a}^{b} f^{p}(t) \mathrm{d} t\right)^{1 / p}, p \neq 0, \\
\exp \left(\frac{1}{b-a} \int_{a}^{b} \ln f(t) \mathrm{d} t\right), p=0 .
\end{array}\right.
$$

The Stolarsky mean $E(a, b ; r, s)$ (see [7]) of two positive numbers $a, b$ is given by 


$$
E(a, b ; r, s)=\left\{\begin{array}{cc}
\left(\frac{r}{s} \cdot \frac{a^{s}-b^{s}}{a^{r}-b^{r}}\right)^{1 /(s-r)}, & r s(s-r)(b-a) \neq 0, \\
\left(\frac{1}{r} \cdot \frac{a^{r}-b^{r}}{\ln a-\ln b}\right)^{1 / r}, & s=0, r(b-a) \neq 0 \\
\mathrm{e}^{-\frac{1}{r}\left(\frac{a^{a^{r}}}{b^{b^{r}}}\right)^{1 /\left(a^{r}-b^{r}\right)},} & r=s, r(b-a) \neq 0 \\
\sqrt{a b}, & r=s=0, a \neq b \\
a, & a=b .
\end{array}\right.
$$

In [2], following theorem is given.

Theorem 1.1. ([2]) Let $f(x)$ be a positive $r$-con- vex function on $[a, b]$ and $G:[0,1] \rightarrow(-\infty,+\infty)$ is defined by

$$
G(t)=\left\{\begin{array}{l}
\left\{\frac{1}{b-a} \int_{a}^{b}\left[\frac{x-a}{b-a} f^{r}(t b+(1-t) x)+\frac{b-x}{b-a} f^{r}(t a+(1-t) x)\right]^{p / r} \mathrm{~d} x\right\}^{1 / p}, r \neq 0, p \neq 0, \\
\left\{\frac{1}{b-a} \int_{a}^{b}\left[f^{\frac{x-a}{b-a}}(t b+(1-t) x) f^{\frac{b-x}{b-a}}(t a+(1-t) x)\right]^{p} \mathrm{~d} x\right\}^{1 / p}, r=0, p \neq 0, \\
\exp \left\{\frac{1}{b-a} \int_{a}^{b} \ln \left[\frac{x-a}{b-a} f^{r}(t b+(1-t) x)+\frac{b-x}{b-a} f^{r}(t a+(1-t) x)\right]^{1 / r} \mathrm{~d} x\right\}^{1 / p}, r \neq 0, p=0, \\
\exp \left\{\frac{1}{b-a} \int_{a}^{b}\left[f^{\frac{x-a}{b-a}}(t b+(1-t) x) f^{\frac{b-x}{b-a}}(t a+(1-t) x)\right] \mathrm{d} x\right\}, r=p=0 .
\end{array}\right.
$$

Then

(i) $G(t)$ is monotonically increasing on $[0,1]$;

(ii) $G(0)=M_{p}(f), G(1)=E(f(a), f(b) ; r, p+r)$.

In [4], following theorems are given.

Theorem 1.2. ([3]) Let $f:[a, b] \rightarrow(0, \infty)$ be $r$-convex function on $[a, b]$ with $a<b$. Then the following inequality holds for $0<r \leq 1$,

$$
\frac{1}{b-a} \int_{a}^{b} f(x) \mathrm{d} x \leq\left(\frac{r}{r+1}\right)^{1 / r}\left[f^{r}(a)+f^{r}(b)\right]^{1 / r} .
$$

Theorem 1.3. ([3]) Let $f, g:[a, b] \rightarrow(0, \infty)$ be $r_{1}$ convex and $r_{2}$-convex functions respectively on $[a, b]$ with $a<b$ Then the following inequality holds for $0<r_{1}, r_{2} \leq 2$,

$$
\frac{1}{b-a} \int_{a}^{b} f(x) g(x) \mathrm{d} x \leq \frac{1}{2}\left(\frac{r_{1}}{r_{1}+2}\right)^{2 / r_{1}} \cdot\left(f^{\prime \prime}(a)+f^{\prime \prime}(b)\right)^{2 / r_{1}}+\frac{1}{2}\left(\frac{r_{2}}{r_{2}+2}\right)^{2 / r_{2}}\left(g^{r_{2}}(a)+g^{r_{2}}(b)\right)^{2 / r_{2}} .
$$

Theorem 1.4. ([3]) Let $f, g:[a, b] \rightarrow(0, \infty)$ be $r_{1}$ convex and $r_{2}$-convex functions respectively on $[a, b]$ with $a<b$ Then the following inequality holds

$$
\begin{aligned}
& \frac{1}{b-a} \int_{a}^{b} f(x) g(x) \mathrm{d} x \\
& \leq\left(\frac{f^{r_{1}}(a)+f^{r_{1}}(b)}{2}\right)^{1 / r_{1}}\left(\frac{g^{r_{2}}(a)+g^{r_{2}}(b)}{2}\right)^{1 / r_{2}}
\end{aligned}
$$

for $r_{1}>1$ and $\frac{1}{r_{1}}+\frac{1}{r_{2}}=1$.

\section{Main Results}

In this paper we obtain some new Hermite-Hadamard type integral inequalities for $r$-convex functions and improve the results of Theorems 1.2-1.4.

The following are extensions of Hermite-Hadamard type inequality:

Theorem 2.1. Let $f:[a, b] \subseteq[0, \infty) \rightarrow(0, \infty)$ be $r$ convex function on $[a, b]$ with $a<b, r \in(-\infty,+\infty)$. Then

$$
\frac{1}{b-a} \int_{a}^{b} f(x) \mathrm{d} x \leq E(f(a), f(b) ; r, r+1) .
$$


Proof. Let $x=t a+(1-t) b, 0 \leq t \leq 1$, then

$$
\frac{1}{b-a} \int_{a}^{b} f(x) \mathrm{d} x=\int_{0}^{1} f(t a+(1-t) b) \mathrm{d} t .
$$

If $f(a)=f(b)$, by the $r$-convexity of $f$, we have

$$
f(t a+(1-t) b) \leq f(a)=f(b)
$$

for any $0 \leq t \leq 1$. So the conclusion is valid.

If $f(a) \neq f(b)$, we have to discuss three cases as following:

Case 1. If $r=-1$, we have

$$
f(t a+(1-t) b) \leq\left[t f^{-1}(a)+(1-t) f^{-1}(b)\right]^{-1}
$$

for any $0 \leq t \leq 1$. Hence, we obtain

$$
\begin{aligned}
& \int_{0}^{1} f(t a+(1-t) b) \mathrm{d} t \leq \int_{0}^{1}\left[t f^{-1}(a)+(1-t) f^{-1}(b)\right]^{-1} \mathrm{~d} t \\
& =\frac{\ln f^{-1}(a)-\ln f^{-1}(b)}{f^{-1}(a)-f^{-1}(b)}=E(f(a), f(b) ;-1,0) .
\end{aligned}
$$

Case 2. If $r=0$, we have

$$
f(t a+(1-t) b) \leq f^{t}(a) f^{1-t}(b)
$$

for any $0 \leq t \leq 1$. Hence, we obtain

$$
\int_{0}^{1} f(t a+(1-t) b) \mathrm{d} t \leq \int_{0}^{1} f^{t}(a) f^{1-t}(b) \mathrm{d} t=\frac{f(a)-f(b)}{\ln f(a)-\ln f(b)}=E(f(a), f(b) ; 0,1) .
$$

Case 3. If $r \neq 0, r \neq-1$, we have

$$
f(t a+(1-t) b) \leq\left[t f^{r}(a)+(1-t) f^{r}(b)\right]^{1 / r}
$$

for any $0 \leq t \leq 1$. Hence, we get

$$
\begin{aligned}
\int_{0}^{1} f(t a+(1-t) b) \mathrm{d} t & \leq \int_{0}^{1}\left[t f^{r}(a)+(1-t) f^{r}(b)\right]^{1 / r} \mathrm{~d} t=\frac{r}{r+1} \frac{f^{r+1}(a)-f^{r+1}(b)}{f^{r}(a)-f^{r}(b)} \\
& =E(f(a), f(b) ; r, r+1) .
\end{aligned}
$$

The proof of Theorem 2.1 is complete.

Corollary 2.1.1. If $r=1$ in Theorem 2.1, we have

$$
\frac{1}{b-a} \int_{a}^{b} f(x) \mathrm{d} x \leq \frac{f(a)+f(b)}{2} .
$$

Theorem 2.2. Let

$$
f, g:[a, b] \subseteq[0, \infty) \rightarrow(0, \infty)
$$

be $r_{1}$-convex and $r_{2}$-convex functions respectively on $[a, b]$ with $a<b, \quad r_{1}, r_{2} \in(-\infty,+\infty)$. Then the following inequality holds

$$
\frac{1}{b-a} \int_{a}^{b} f(x) g(x) \mathrm{d} x \leq\left[E\left(f^{p}(a), f^{p}(b) ; \frac{r_{1}}{p}, \frac{r_{1}}{p}+1\right)\right]^{1 / p} \times\left[E\left(g^{q}(a), g^{q}(b) ; \frac{r_{2}}{q}, \frac{r_{2}}{q}+1\right)\right]^{1 / q}
$$

for any $p, q>0$ and $\frac{1}{p}+\frac{1}{q}=1$.

Proof. Let $x=t a+(1-t) b, 0 \leq t \leq 1$, then we have $\frac{1}{b-a} \int_{a}^{b} f(x) g(x) \mathrm{d} x=\int_{0}^{1} f(t a+(1-t) b) g(t a+(1-t) b) \mathrm{d} t$.

If $f(a) \neq f(b), g(a) \neq g(b)$, then

1) when $r_{1} r_{2} \neq 0$, by the $r_{1}$-convexity and $r_{2}$-con- vexity of functions $f, g$ respectively, we have

$$
f(t a+(1-t) b) \leq\left[t f^{n}(a)+(1-t) f^{n}(b)\right]^{1 / n}
$$

and

$$
g(t a+(1-t) b) \leq\left[\operatorname{tg}^{r_{2}}(a)+(1-t) g^{r_{2}}(b)\right]^{1 / r_{2}}
$$

for any $0 \leq t \leq 1$. So we obtain

$$
\begin{aligned}
\frac{1}{b-a} \int_{a}^{b} f(x) g(x) \mathrm{d} x & =\int_{0}^{1} f(t a+(1-t) b) g(t a+(1-t) b) \mathrm{d} t \\
& \leq \int_{0}^{1}\left[t f^{\prime \prime}(a)+(1-t) f^{\prime \prime}(b)\right]^{1 / 11} \cdot\left[\operatorname{tg}^{r_{2}}(a)+(1-t) g^{r_{2}}(b)\right]^{1 / r_{2}} \mathrm{~d} t
\end{aligned}
$$

By the Hölder's integral inequality and Theorem 2.1, we have 


$$
\begin{aligned}
& \int_{0}^{1}\left[t f^{r_{1}}(a)+(1-t) f^{r_{1}}(b)\right]^{1 / r_{1}} \cdot\left[\operatorname{tg}^{r_{2}}(a)+(1-t) g^{r_{2}}(b)\right]^{1 / r_{2}} \mathrm{~d} t \\
& \leq\left(\int_{0}^{1}\left[t f^{r_{1}}(a)+(1-t) f^{r_{1}}(b)\right]^{p / r_{1}} \mathrm{~d} t\right)^{1 / p} \cdot \int_{0}^{1}\left(\left[t g^{r_{2}}(a)+(1-t) g^{r_{2}}(b)\right]^{q / r_{2}} \mathrm{~d} t\right)^{1 / q} \\
& =\int_{0}^{1}\left(\left[t\left[f^{p}(a)\right]^{\eta^{1 / p}}+(1-t)\left[f^{p}(b)\right]^{\eta^{1 / p}}\right]^{p / \eta} \mathrm{d} t\right)^{1 / p} \cdot \int_{0}^{1}\left(\left[t\left[g^{q}(a)\right]^{r_{2} / q}+(1-t)\left[g^{q}(b)\right]^{r_{2} / q}\right]^{q / r_{2}} \mathrm{~d} t\right)^{1 / q} \\
& =\left[E\left(f^{p}(a), f^{p}(b) ; \frac{r_{1}}{p}, \frac{r_{1}}{p}+1\right)\right]^{1 / p} \times\left[E\left(g^{q}(a), g^{q}(b) ; \frac{r_{2}}{q}, \frac{r_{2}}{q}+1\right)\right]^{1 / q} .
\end{aligned}
$$

2) when $r_{1} r_{2}=0$, we just prove for $r_{1}=0, r_{2} \neq 0$ which is similar to $r_{1}=r_{2}=0$ and $r_{1} \neq 0, r_{2}=0$. By the Hölder's integral inequality, Theorem 2.1 and $r_{1}$-convexity and $r_{2}$-convexity of functions $f, g$ respectively, we have

$$
\begin{aligned}
& \frac{1}{b-a} \int_{a}^{b} f(x) g(x) \mathrm{d} x \\
& =\int_{0}^{1} f(t a+(1-t) b) g(t a+(1-t) b) \mathrm{d} t \\
& \leq \int_{0}^{1} f^{t}(a) f^{1-t}(b)\left[\operatorname{tg}^{r_{2}}(a)+(1-t) g^{r_{2}}(b)\right]^{1 / r_{2}} \mathrm{~d} t \\
& \leq\left(\int_{0}^{1} f^{p t}(a) f^{p(1-t)}(b) \mathrm{d} t\right)^{1 / p} \\
& \cdot\left(\int_{0}^{1}\left[t g^{r_{2}}(a)+(1-t) g^{r_{2}}(b)\right]^{q / r_{2}} \mathrm{~d} t\right)^{1 / q} \\
& =\left[E\left(f^{p}(a), f^{p}(b) ; 0,1\right)\right]^{1 / p} \\
& \cdot\left[E\left(g^{q}(a), g^{q}(b) ; \frac{r_{2}}{q}, \frac{r_{2}}{q}+1\right)\right]^{1 / q} .
\end{aligned}
$$

If $f(a)=f(b)$ or $g(a)=g(b)$, by Theorem 2.1 we obtain the conclusion, which the proof of Theorem 2.2 is completed.

Corollary 2.2.1. Under the conditions of Theorem 2.2, if $\frac{1}{r_{1}}+\frac{1}{r_{2}}=1$ for any $r_{1}, r_{2}>0$, then we have

$$
\begin{aligned}
& \frac{1}{b-a} \int_{a}^{b} f(x) g(x) \mathrm{d} x \\
& \leq\left(\frac{f^{n_{1}}(a)+f^{n_{1}}(b)}{2}\right)^{1 / n}\left(\frac{g^{r_{2}}(a)+g^{r_{2}}(b)}{2}\right)^{1 / r_{2}} .
\end{aligned}
$$

In particular, if $r_{1}=r_{2}=2$, then we have

$$
\begin{aligned}
& \frac{1}{b-a} \int_{a}^{b} f(x) g(x) \mathrm{d} x \\
& \leq\left(\frac{f^{2}(a)+f^{2}(b)}{2}\right) \cdot\left(\frac{g^{2}(a)+g^{2}(b)}{2}\right) .
\end{aligned}
$$

If $f(a)=f(b), g(a)=g(b)$, we have

$$
\frac{1}{b-a} \int_{a}^{b} f(x) g(x) \mathrm{d} x \leq f(a) g(a)
$$

Corollary 2.2.2. Under conditions of Theorem 2.2, if $f(x)=g(x)$ and $r_{1}=r_{2}$ then we have

$\frac{1}{b-a} \int_{a}^{b} f(x) g(x) \mathrm{d} x \leq E^{2}\left(f(a), f(b) ; r_{1}, 2+r_{1}\right)$.

In particular, if $r_{1}=r_{2}=0$, then we have

$$
\frac{1}{b-a} \int_{a}^{b} f^{2}(x) \mathrm{d} x \leq \frac{f^{2}(a)+f^{2}(b)}{2}
$$

Theorem 2.3. Let $f, g:[a, b] \subseteq[0, \infty) \rightarrow(0, \infty)$, $f g \in L[a, b], \quad r_{1}, r_{2} \in(-\infty,+\infty)$ and $f^{p}(x), g^{q}(x)$ be $r_{1}$-convex and $r_{2}$-convex functions respectively on $[a, b]$ with $a<b$. Then the following inequality holds

$$
\begin{aligned}
& \frac{1}{b-a} \int_{a}^{b} f^{2}(x) \mathrm{d} x \leq {\left[E\left(f^{p}(a), f^{p}(b) ; r_{1}+r_{2}+1\right)\right] } \\
& \cdot {\left[E\left(g^{q}(a), g^{q}(b)\right) ; r_{1}+r_{2}+1\right]^{1 / q} } \\
& \text { for any } p, q>0 \text { and } \frac{1}{p}+\frac{1}{q}=1 .
\end{aligned}
$$

Proof. Let $x=t a+(1-t) b, 0 \leq t \leq 1$, then we have $\frac{1}{b-a} \int_{a}^{b} f(x) g(x) \mathrm{d} x=\int_{0}^{1} f(t a+(1-t) b) g(t a+(1-t) b) \mathrm{d} t$.

By the Hölder's integral inequality, Theorem 2.1 and $r_{1}$-convexity and $r_{2}$-convexity of function $f^{p}(x), g^{q}(x)$ respectively, we have

$$
\begin{aligned}
& \frac{1}{b-a} \int_{a}^{b} f(x) g(x) \mathrm{d} x \\
& =\int_{0}^{1} f(t a+(1-t) b) g(t a+(1-t) b) \mathrm{d} t \\
& \leq\left(\int_{0}^{1} f^{p}(t a+(1-t) b) \mathrm{d} t\right)^{1 / p} \\
& \cdot\left(\int_{0}^{1} g^{q}(t a+(1-t) b) \mathrm{d} t\right)^{1 / q} \\
& \leq\left[E\left(f^{p}(a), f^{p}(b) ; r_{1}, r_{1}+1\right)\right]^{1 / p} \\
& \cdot\left[E\left(g^{q}(a), g^{q}(b) ; r_{2}, r_{2}+1\right)\right]^{1 / q}
\end{aligned}
$$

This completed the proof of Theorem 2.3.

Corollary 2.3.1. Under the conditions of Theorem 2.3, 
if $p=q=2$ and $r_{1}=r_{2}=\frac{1}{2}$, then we have

$$
\begin{aligned}
& \frac{1}{b-a} \int_{a}^{b} f(x) g(x) \mathrm{d} x \\
& \leq \sqrt{\frac{f^{2}(a)+f(a) f(b)+f^{2}(b)}{3}} \\
& \sqrt{\frac{g^{2}(a)+g(a) g(b)+g^{2}(b)}{3}} .
\end{aligned}
$$

In particular, if $f(a)=f(b), g(a)=g(b)$, we have

$$
\frac{1}{b-a} \int_{a}^{b} f(x) g(x) \mathrm{d} x \leq f(a) g(a)
$$

In this paper, we obtained three new Hermite-Hadamard type integral inequalities for $r$-convex functions, which improved the results of Theorems 1.2-1.4 by Hölder's integral inequality, Stolarsky mean and convexity of function. The special case of new Hermite-Hadamard type integral inequalities is classical Hermite-Hadamard type integral inequality. So it improved the classical one.

\section{Acknowledgements}

The first author was supported in part by the National Natural Science Foundation of China under Grant No. 11161033 and Inner Mongolia Natural Science of China under Grant No. 2010MS0119.

\section{REFERENCES}

[1] C. E. M. Pearce, J. Peccaric and V. Simic, "Stolarsky Means and Hadamard's Inequality," Journal of Mathematical Analysis and Applications, Vol. 220, No. 1, 1998, pp. 99-109. doi:10.1006/jmaa.1997.5822

[2] G.-S. Yang, "Refinements of Hadamard's Inequality for $r$-Convex Functions," Indian Journal of Pure and Applied Mathematics, Vol. 32, No. 10, 2001, pp. 1571-1579.

[3] N. P. N. Ngoc, N. V. Vinh and P. T. T. Hien, "Integral Inequalities of Hadamard Type for $r$-Convex Functions," International Mathematical Forum, Vol. 4, No. 35, 2009, pp. 1723-1728.

[4] M. K. Bakula, M. E. Özdemir and J. Pečarić, "Hadamard Type Inequalities for $m$-Convex and $(\alpha-m)$-Convex Functions," Journal of Inequalities in Pure and Applied Mathematics, Vol. 9, No. 4, 2008, Article ID: 96.

[5] P. M. Gill, C. E. M. Pearce and J. Pečarić, "Hadamard's Inequality for $r$-Convex Functions," Journal of Mathematical Analysis and Applications, Vol. 215, No. 2, 1997. pp. 461-470. doi:10.1006/jmaa.1997.5645

[6] A. G. Azpeitia, "Convex Functions and the Hadamard Inequality," Revista Colombiana de Matemáticas, Vol. 28, No. 1, 1994, pp. 7-12.

[7] K. B. Stolarsky, "Generalizations of the Logarithmic Mean," Mathematics Magazine, Vol. 48, No. 2, 1975, pp. 87-92. doi: $10.2307 / 2689825$ 